\title{
"Food Offerings": A Major Factor Impeding Adherence with Weight Loss Diets in Overweight and Obese Individuals
}

\section{Reza Rastmanesh ${ }^{1 *}$ and Marci E Gluck ${ }^{2}$}

${ }^{1}$ Clinical Nutrition and Dietetics Department, Shahid Beheshti University of Medical Sciences, National Nutrition and Food Sciences Technology Research Institute, Tehran, Iran

${ }^{2}$ Obesity and Diabetes Clinical Research Section, Phoenix Epidemiology and Clinical Research Branch, National Institute of Diabetes and Digestive and Kidney

Diseases, National Institutes of Health, Phoenix, Arizona, USA

\begin{abstract}
The effect of food offerings and other impeding factors on dietary adherence with weight loss and their relationship to self-esteem was examined. 2496 overweight and obese dieting males and females were recruited from aerobic health clubs in Tehran and completed a battery of questionnaires. Respondents rated 7 impeding factors to dietary adherence on a 5-point (0-4) Likert scale and completed the Rosenberg Self-Esteem Scale. Participants were categorized as successful weight losers $(73 \%)$ and weight regainers $(27 \%)$. Weight regainers were most impeded by food offerings, low calorie dietary recommendations, lack of vigilance with regard to weight control, use of eating to regulate mood, and failure to achieve weight goal. Successful weight losers were most impeded by dissatisfaction with weight achieved, failure to achieve weight goal, and lack of dietary preferences. Moreover, successful weight losers were more than three times more likely to reject a food offering while dieting $(p<0.001)$ and scored significantly higher on the measure of self-esteem compared to weight regainers $(p<0.0001)$. The acceptance of food offerings was the most influential impeding factor to adherence with weight loss diets in a group of weight regainers. Moreover, the acceptance of food offerings was related to lower self-esteem. Findings from this study suggest that helping patients, change responses to food offerings and other socially motivating reinforcers as well as improving selfesteem should be featured components of behavioral weight loss interventions.
\end{abstract}

Keywords: Food offerings; Self-esteem; Obesity; Weight regain

\section{Introduction}

Failure to adhere to weight loss diets is associated with failure to achieve weight goals, dissatisfaction with the weight achieved, the tendency to evaluate self-worth in terms of weight and shape, a lack of vigilance with regard to weight control, and the tendency to use eating to regulate mood [1]. Even when people are successful at losing weight, the long-term outcome for the vast majority is that they regain the weight they lost [2].

Mounting evidence suggests that biological mechanisms including a decrease in energy expenditure during dieting and compensatory adaptations that would be expected to increase appetite, contribute substantially to relapse from weight loss during dietary therapy [35]. However, because most individuals are frequently bombarded by an abundance of attractive food cues, it is likely that food availability also plays a significant role in dietary non-adherence [6]. As Brownell and Horgen has pointed out, the super-abundance of food cues in our society has created a "toxic environment" that promotes overeating and overweight rather than under-eating and weight loss [7].

Research demonstrates that the presence of external food cues makes restriction of intake more difficult. Exposure to attractive food cues increases food consumption in humans $[8,9]$ even when they are already satiated $[10,11]$. In fact, the presence of food cues can be more potent than signals of satiety, [11] and heightened food-cue reactivity may be a risk factor for overeating and weight gain [12].

The offering of food to friends and guests is a prevalent and institutionalized habit in most cultures, and being polite is culturally important in many societies. As a result many people accept food offers as a show of politeness and respect, even when they are dieting to lose weight. The decision to reject or accept a food offer might also be highly influenced by individual personality characteristics. In particular, obesity is associated with lower self-esteem $[13,14]$ and people with higher self-esteem show better adherence to weight loss diets [15]. The acceptance of food offerings therefore, might be a significant risk factor for dietary non-adherence and/or relapse following successful weight loss, yet this construct has not yet been addressed in the scientific literature.

It was therefore aimed to identify factors leading to dietary nonadherence, including the acceptance or rejection of food offerings. Specifically, it was hypothesized that non-adherers to a weight loss diet (weight regainers) would be more likely to accept food offerings while dieting and would score lower on a measure of self-esteem.

\section{Methods}

\section{Participants}

This was a cross-sectional study conducted in 2008 in Tehran, the major cosmopolitan center and capital of Iran. Three thousand prepaid envelopes containing a battery of questionnaires were distributed to male and female members from 52 randomly selected registered aerobic health clubs. Inclusion criteria were willingness to participate in the study, a history of dieting and membership in an aerobic health club during the last 24 months, body mass index (BMI) $>24.9 \mathrm{~kg} / \mathrm{m}^{2}$ at the time of registration to the club, and engaging in a regular exercise program (at least 2times/week). Informed consent was obtained from all participants after the nature of the procedures had been fully

*Corresponding author: Reza Rastmanesh, Clinical Nutrition and Dietetics Department, Shahid Beheshti University of Medical Sciences, National Nutrition and Food Sciences Technology Research Institute, Tehran, Iran, E-mail: rezar@sbmu.ac.ir

Received October 25, 2012; Published November 22, 2012

Citation: Rastmanesh R, Gluck ME (2013) "Food Offerings": A Major Factor Impeding Adherence with Weight Loss Diets in Overweight and Obese Individuals. J Nutr Disorders Ther 3: 122. doi:10.4172/2161-0509.1000122

Copyright: ( 2013 Rastmanesh R, et al. This is an open-access article distributed under the terms of the Creative Commons Attribution License, which permits unrestricted use, distribution, and reproduction in any medium, provided the original author and source are credited. 
explained and participants were assured anonymity. The study was approved by the Ethics Committee of SBMU and the study protocol conformed to the ethical guidelines of the Declaration of Helsinki.

\section{Measures}

Demographics: Weight was measured at the club by a standard scale to the nearest 100 grams while subjects were wearing as little clothing as possible. Height was measured with a standard scale for height (Seca, 206) to the nearest millimeter. Previous weight and height had also been assessed and recorded in the club's registration book two years prior to the beginning of the study by same gender person (time zero). Participants were dummy-coded as weight losers or weight regainers based on the following criteria:

Successful weight losers: Participants with a history of overweight (BMI $>24.9 \mathrm{~kg} / \mathrm{m}^{2}$ ) or obesity (BMI $>29.9 \mathrm{~kg} / \mathrm{m}^{2}$ ) who, at some point within the last 2 years, had lost at least $10 \%$ of their initial body weight as a result of deliberate caloric restriction and aerobic exercise, and who had maintained their new lower weight (to within a range of 3.2 $\mathrm{kg}$ ) for at least 1 year. This range discriminates between mere weight fluctuation and weight regain, and ensures a clear demarcation between weight losers and weight regainers [1].

Weight regainers: Participants meeting the same criteria as the weight losers except that they had regained weight to within $3.2 \mathrm{~kg}$ of their original body weight [1].

Self-esteem: The Rosenberg Self-Esteem Scale (RSES; Persian standardized, and validated version) [16] measures global self-esteem and personal worthlessness. Higher scores indicate higher self-esteem [17].

Impeding factors to dietary adherence: Participants were asked to rate, on Likert scales from 0 (not important at all) to 4 (very important), the most important impeding factors to adhering with their weight loss diets. Answers included "low calorie dietary recommendations," "food offerings," "lack of dietary preferences," "failure to achieve weight goal," "dissatisfaction with the weight achieved," "lack of vigilance with regard to weight control," and "using eating to regulate mood."

Participants were also asked "what would you do if you were offered food, a heavy calorie sweet, or a snack (by parents, spouse or a friend) at any time (morning, evening, dinner, snack) while you were dieting?" Answers included: reject food offer, accept food offer or cannot decide. In addition, participants were asked to write a statement in response to an open-ended question: "why would you accept a food offer while you are on a diet?" A qualitative analysis was performed in order to identify themes describing the reasons why dieting people accept food offerings. The psychometric properties of this questionnaire assessing the impeding factors to dietary adherence were validated in terms of face content, construct and criterion validity and test-retest reliability in a convenience sample of 41 Iranians aged 12-59 years in a previous study (Cronbach alpha of 0.93 and construct validity of 0.96 and testretest reliability 0.92 ).

Socioeconomic status: It was measured by self-reported household income and education level. Membership to an aerobic club in Tehran usually entails a high or mid-high socioeconomic status and education.

Data analysis: All returned surveys were included in the analysis. Incomplete or missing data are reported as such. Descriptive statistics, including medians and ranges, were calculated for each item. Chisquare tests were used for categorical variables. The normality of all variables was assessed by examining their normal plots and using
Kolmogorov-Smirnov test. Since data had non-normal distributions Kruskal-Wallis and Mann-Whitney U tests were used.

Differences in mean self-esteem scores between weight losers and regainers were assessed using Mann-Whitney U. All tests were 2-tailed, and $\mathrm{p}<0.05$ was considered significant. Data were analyzed with SPSS version 17 (SPSS Inc, Chicago).

\section{Results \\ Participants}

The response rate was $88.1 \%$. The characteristics of the two groups are presented in table 1 . At time zero, there were no significant differences between those in the successful weight losers versus the weight regainers groups on any demographic variable including marital status, age, BMI, gender, and socioeconomic status (Table 1). After an average of 2 years of being on a weight loss diet and engaging in a regular exercise, BMI was significantly higher in the weight regainer group compared to the successful weight loser group (28.5 \pm 3.3 vs. $\left.24.9 \pm 3.3 \mathrm{~kg} / \mathrm{m}^{2}, \mathrm{df}=2641, \mathrm{p}<0.001\right)$. Weight regainers and successful weight losers were not evenly distributed across these 52 health clubs $\left(\chi^{2}=2573 ; \mathrm{df}=51, \mathrm{p}=0.001\right)$. However, successful weight losers reported similar number of hours per week engaging in regular exercise program compared to the weight regainers $(4.9 \pm 0.7$ vs. $4.8 \pm 0.8$, respectively; $\mathrm{df}=2, \mathrm{NS})$.

\section{Impeding factors to dietary adherence during weight loss}

Table 2 illustrates the relative importance of the factors impeding adherence in the two groups. There were significant differences between the two subgroups for all 7 impeding factors, and also in the responses to the qualitative question regarding the acceptance of food offerings (Mann-Whitney $\mathrm{U}, \mathrm{df}=2$, all p's $<0.001$ ). Successful weight

\begin{tabular}{|c|c|c|}
\hline & Weight losers & Weight regainers \\
\hline No (\%) & 1779 (718.9) & $717(28.2)$ \\
\hline \multicolumn{3}{|l|}{ Marital Status $¥$} \\
\hline \multicolumn{3}{|l|}{ Single } \\
\hline Male & $80(10.2)$ & $44(12.6)$ \\
\hline Female & $706(89.8)$ & $306(87.4)$ \\
\hline \multicolumn{3}{|l|}{ Married } \\
\hline Male & $87(12.4)$ & $23(9.0)$ \\
\hline Female & $616(87.6)$ & $222(91.0)$ \\
\hline \multicolumn{3}{|l|}{ Divorced } \\
\hline Male & $11(47.8)$ & $5(50)$ \\
\hline Female & $12(52.2)$ & $5(50)$ \\
\hline \multicolumn{3}{|l|}{ Unknown } \\
\hline Male & $26(9.8)$ & $8(7.2)$ \\
\hline Female & $241(90.2)$ & $104(92.8)$ \\
\hline \multicolumn{3}{|l|}{ Gender } \\
\hline Male & 204 (11.5) & $80(11.2)$ \\
\hline Female & $1575(88.5)$ & $637(88.8)$ \\
\hline Age $^{\ddagger}$ & $29.75 \pm 9.0$ & $29.55 \pm 9.20$ \\
\hline Body Mass Index & $29.17 \pm 3.60$ & $28.95 \pm 3.30$ \\
\hline \multicolumn{3}{|l|}{ Socioeconomic Status } \\
\hline High & $648(36.4)$ & $287(40)$ \\
\hline Medium-High & $1131(63.6)$ & $430(60)$ \\
\hline
\end{tabular}

${ }^{*} \mathrm{n}=2496$

¥¥Marital status was analyzed by 4 levels (single, married, divorced, unknown) to the levels of weight

$\ddagger$ Data are Mean \pm SD

There were no significant differences between the groups on any variable.

Table 1: Characteristics of subjects* 
Citation: Rastmanesh R, Gluck ME (2013) "Food Offerings": A Major Factor Impeding Adherence with Weight Loss Diets in Overweight and Obese Individuals. J Nutr Disorders Ther 3: 122. doi:10.4172/2161-0509.1000122

Page 3 of 5

\begin{tabular}{|c|c|c|c|c|c|c|c|}
\hline \multicolumn{8}{|c|}{ Level of influence on failure (and numeric value):Percent of respondents } \\
\hline & None & Minor & Moderate & Major & Maximum & Median & $\begin{array}{c}\text { Interquartile } \\
\text { Range }\end{array}$ \\
\hline Factor ${ }^{* a}$ & (0) & (1) & (2) & & (3) & (4) & \\
\hline \multicolumn{8}{|l|}{ Weight regainers } \\
\hline Food offerings ${ }^{b}$ & $2(2.0,0)$ & $2.9(1.8,1.1)$ & $7.3(6.0,1.3)$ & $35.4(0.3,35.1)$ & $52.4(1.1,51.3)$ & 4 & $1-4$ \\
\hline Low calorie dietary recommendations ${ }^{b}$ & $3.1(2.4,0.7)$ & $2.9(1.8,1.1)$ & $6.1(5.5,0.6)$ & $41.6(0.4,41.4)$ & $46.3(1,45.3)$ & 3 & $1-4$ \\
\hline Lack of vigilance with regard to weight control ${ }^{b}$ & $8.1(7.0,1.1)$ & $3.1(1.8,1.3)$ & $1.1(1.0,0.1)$ & $41.6(0.4,41.1)$ & $46.2(1.0,45.2)$ & 3 & $1-4$ \\
\hline Use eating to regulatemood & $4.3(0.1,4.2)$ & $2.9(0.3,2.6)$ & $0.8(0,0.8)$ & $78.5(8.4,70.2)$ & $13.4(2.4,11.0)$ & 3 & $0-4$ \\
\hline \multicolumn{8}{|l|}{ Lack of dietary } \\
\hline preference & $0.6(0,0.6)$ & $22.6(2.4,20.2)$ & & $17.9(2.0,15.9)$ & & $55.5(6.3,49.2)$ & $3.5(0.6,2.9)$ \\
\hline Failure to achieve weight goalb & $39.1(4.4,34.7)$ & $29.3(2.9,26.4)$ & $5.7(2.1,3.6)$ & $16(0.8,15.2)$ & $9.9(1.0,8.9)$ & 1 & 3-4 \\
\hline Dissatisfaction with the weight achieved & $34(3.3,30.7)$ & $20.2(2.6,17.6)$ & & $9.2(0.6,8.6)$ & $35.8(4.6,31.2)$ & $0.7(0,0.7)$ & \\
\hline \multicolumn{8}{|l|}{ Weight losers } \\
\hline Food offerings ${ }^{b}$ & $0.3(0.2,0.1)$ & $48.1(0.5,47.6)$ & $44.5(11.2,33.4)$ & $6.3(0.9,5.4)$ & & $0.7(0.4,0.3)$ & 2 \\
\hline Dissatisfaction with the weight achieved & $0.8(0.1,0.7)$ & $33.1(4.3,28.8)$ & $30.4(3.7,26.6)$ & $6.5(1.0,5.6)$ & $29.2(4.1,25.1)$ & 2 & 3-4 \\
\hline Failure to achieve weight goal ${ }^{b}$ & $0.5(0.2,0.3)$ & $39.1(0.6,38.5)$ & $42.7(10.5,32.2)$ & $7.8(1.0,6.9)$ & $9.9(0.9,8.9)$ & 2 & $1-4$ \\
\hline Lack of dietary preferences & $4.6(0.4,4.2)$ & $64.8(8.7,56.1)$ & $20(2.8,17.2)$ & $6.2(0.7,5.6)$ & $4.4(0.6,3.7)$ & 1 & $1-4$ \\
\hline Use eating to regulate mood & $22.4(3.2,19.2)$ & $57.6(7.5,50.1)$ & $11.2(1.6,9.6)$ & $7.1(0.7,6.4)$ & $1.7(0.3,1.4)$ & 1 & $0-4$ \\
\hline \multicolumn{8}{|l|}{ Low calorie dietary } \\
\hline recommendations ${ }^{\mathrm{b}}$ & $45.3(11.1,34.2)$ & $47.2(0.5,46.7)$ & $0.6(0,0.6)$ & $6.6(1.2,5.3)$ & $0.4(0.3,0.1)$ & 1 & $1-4$ \\
\hline Lack of vigilance with regard to weight controlb & $44.7(11.2,33.5)$ & $48.5(0.8,47.7)$ & $0.1(0,0.1)$ & $6.4(1.2,5.2)$ & & $0.3(0,0.3)$ & 1 \\
\hline
\end{tabular}

Data are percentages and as Total $(\mathrm{M}, \mathrm{F})$.

*In descending order of influence.

${ }^{a}$ There were significant differences between two subgroups on all 7 factors (Mann-Whitney $U, d f=2, p<0.0001$ ).

${ }^{\mathrm{b}}$ There were significant differences between males and females in subgroups (Mann-Whitney $U$, test df $=2, p<0.0001$ ).

There were significantly higher ratings for "food offerings" in the weight regainer group relative to the weight loss group (Mann-Whitney $U$, test $d f=2$, $p<0.0001$ ).

Table 2: Influence of factors impeding compliance with weight loss diet. Variables are percentages and parentheses indicate the male and female percentages.

losers reported being most influenced in their failure to adhere to weight loss diets by dissatisfaction with the weight achieved, failure to achieve weight goal, and lack of dietary preferences. Weight regainers reported being most influenced by food offerings, low calorie dietary recommendations, lack of vigilance with regard to weight control, use of eating to regulate mood, and failure to achieve weight goal (Table 2).

\section{Self-esteem}

The weight regainers scored significantly lower on the measure of self-esteem $(13.5 \pm 0.8)$ compared to subjects in successful weight loser group $(22.9 \pm 4.9)(\mathrm{z}=-39.659, \mathrm{p}<0.0001$, Figure 1$)$, who were also more than three times more likely to reject a food offering while dieting $\left(\chi^{2}=898, p<0.0001\right.$, Figure 2) (Figure 1).

When asked, "would you accept a food offering while dieting," those who reported "no" had significantly higher self-esteem scores $(22.1 \pm 5.4)$ than those who answered they "couldn't decide" (18.2 \pm 5.9) and those who answered "yes" they would accept the food offering $\left(15.6 \pm 4.6, \chi^{2}=462, \mathrm{df}=2, \mathrm{p}<0.0001\right)$.

\section{Gender differences}

Within the successful weight loser group, females were more than 3 times more likely than males to report low calorie dietary recommendations and food offerings as the most influential impeding factors to weight loss $\left(\chi^{2}=447, \mathrm{p}<0.001\right.$ and $\chi^{2}=431, \mathrm{p}<0.001, \mathrm{df}=4$, respectively). Females in that group were also more than 1.3, and more than 5 times to report failure to achieve weight loss goal, and lack of vigilance with regard to weight control $\left(\chi^{2}=204, p<0.001, \chi^{2}=378\right.$, $\mathrm{p}<0.001, \mathrm{df}=4$, respectively) as additional impeding factors. There were no significant differences between males and females in terms of lack of dietary preferences, dissatisfaction with the weight achieved, using eating to regulate mood or responses to a food offering while dieting.

In the weight regainer group, females were more than 5 times more likely than males to report low calorie recommendation and food offerings $\left(\chi^{2}=492, \mathrm{p}<0.001, \mathrm{df}=4\right)$, more than 1.2 , and more than 5 times to report failure to achieve weight loss goal, and lack of vigilance with regard to weight control $\left(\chi^{2}=31.2, p<0.001\right.$, and $\chi^{2}=485, p<0.001$, $\mathrm{df}=4$, respectively) as the most influential impeding factors. There were no significant differences between males and females in terms of lack of dietary preferences, dissatisfaction with the weight achieved, using eating to regulate mood, or responses to a food offering while dieting.

\section{Qualitative analysis}

The qualitative analysis revealed two major themes for accepting a food offering while dieting: food liking and low self-esteem. An example of a weight regainer's written statement was, "if I was offered a food, I would accept foods I like and I would reject food I don't like." Other examples were "When I am gaining weight, I feel lower self-esteem and I am more likely to accept my colleague's food offer in the cafeteria," and "I feel the more you weigh, the faster you quit your weight loss diet." An example of a weight losers written statement was, "if I was offered a food, I would reject the food no matter if I like it or I don't like."

Within the successful weight losers group, $19 \%$ endorsed food liking related themes for accepting food offerings compared to $37 \%$ of those in the weight regainers group $(\mathrm{p}<0.001)$. Significantly more subjects in the successful weight losers group (22\%) indicated that low self-esteem related themes were related to the acceptance of food offerings compared to only $9.5 \%$ of those in the weight regainers group $(\mathrm{p}<0.001)$. 


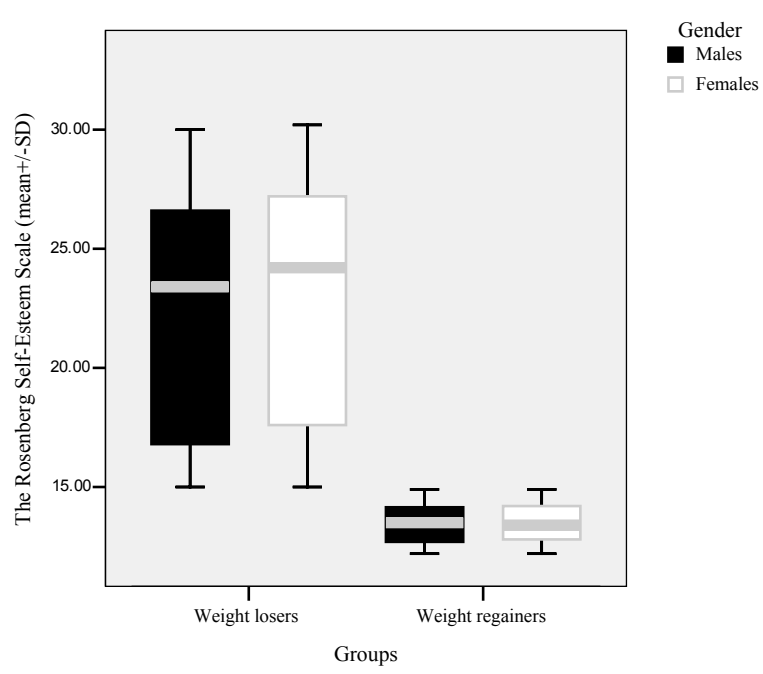

The weight regainers scored significantly lower on the measure of self-esteem compared to subjects in successful weight loser group $(13.5 \pm 0.8,22.9 \pm 4.9$ respectively, $z=-39.569, p<0.0001$, Mann-Whitney $U$ test). Within the weight regainer group, there were no significant difference between males and females, however, females scored significantly higher than males within the weight lose group $(23.0 \pm 4.93,22.2 \pm 4.8$, respectively, $t=-2.44, d f=1918, p<0.01$, T-test $)$.

Figure 1: The weight regainers group had significantly lower self-esteem compared to the weight loser group.

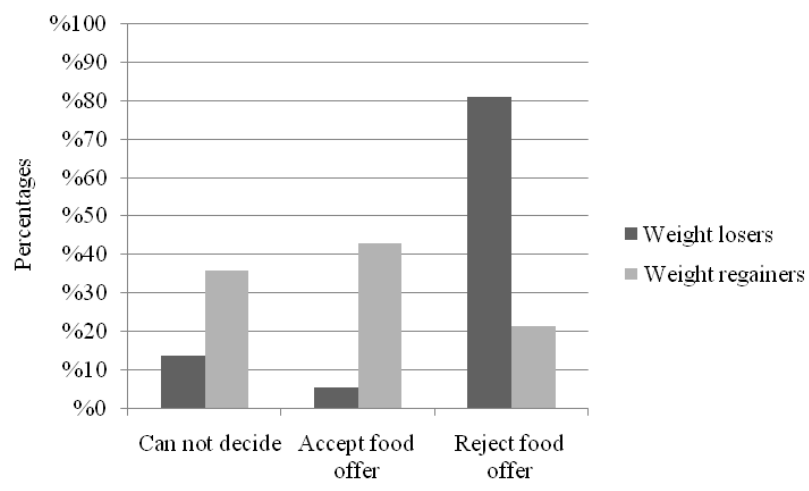

Odds ratio test showed that weight regainers were more than eight times more likely to accept a food offering than successful weight losers while dieting $\left(x^{2}=898, d f=2, p<0.0001\right)$

Figure 2: Responses to the question "what would you do if you were to be offered a food while you are dieting?"

\section{Discussion}

Consistent with our hypotheses, weight regainers rated food offerings as the most influential impeding factor to adherence with a weight loss diet. They were also more likely to accept a food offering while dieting, and scored significantly lower on a measure of self-esteem compared to successful weight losers. Also in our study, females in both the successful weight loss and weight regainer groups were more likely than males to report that food offerings, a low calorie recommendation, failure to achieve weight goal, and lack of vigilance with regard to weight control were impeding factors to dietary adherence to weight loss. These findings suggest that the patterns of psychological behaviors that can impede dietary adherence to weight loss diets are significantly different between successful weight losers and weight regainers, and between males and females.
Recent studies have demonstrated that when in a group setting, an individuals' intake was observed to be strongly influenced by the behavior of others [18] and a strong relationship exists between the amounts of food eaten by members of a dyad [19]. However, participants in these studies did not identify their partners' behavior, but rather hunger and taste as the primary determinants of their own intake. It is possible then, that individuals are unable to accurately identify reasons for eating behaviors and dietary non-adherence. Moreover, it is likely that the acceptance of food offerings is influenced by social behavior but perceived by the recipient to be influenced by hedonics (e.g. involvement of cognitive, reward, and emotional factors) and homeostatic aspects (physical hunger and regulation of energy balance). In particular, the weight regainers in the current study were more likely to endorse food hedonic related themes for accepting food offerings when compared to their successful weight losing counterparts. It is plausible that weight regainers are more responsive to hedonic qualities of food in order to adapt to their lower self-esteem and cope with stressful situations but this has not been directly tested [20].

In most randomized controlled trials in Western countries, men appear to have more successful weight loss outcomes than women [21-23]. Similarly, the men in our study lost more weight compared to women, and women in both the weight loss and weight regainer groups rated food offerings as a greater impeding factor compared to males. It has been previously shown that women who practiced more strict Islamic veiling techniques in Iran had increased body satisfaction and self-esteem, and decreased depression scores and desire for rhinoplasty [24]. It has been also previously reported that women engage in weight loss programs and join health clubs mainly due to non-health related reasons (e.g. to improve body image) whereas men mainly engage in such programs due to health related reasons [24]. As veil practicing and efforts to improve body image are behaviors that impact on how one's appearance is perceived by others, our finding regarding women's increased acceptance of food offerings, which also relates to perception of others, was not surprising. Also, it was observed gender differences in ratings of different impeding factors for dietary non-adherence which suggests that weight control programs should consider such differences between men and women when prescribing weight reduction programs.

There are several limitations to our study. First, the study did not include any measures of eating pathology such as restrained eating or binge eating, both of which might be highly correlated with self-esteem and the acceptance of food offerings. It would be useful to know the frequency of food offerings and the frequency of acceptance of such offers, but this was not assessed. The intensity of physical activity or caloric intake, each of which could independently explain weight gain was also not measured. Also the selection of study participants via aerobic health clubs could make our findings somewhat less generalizable. This was a cross-sectional study and our findings do not imply causality. Lastly, the term "food offerings" might have different meanings and the perceived social pressure to accept such an offer likely differs greatly in different countries. Future studies should further investigate the role of food offerings, the cultural meaning of food acceptance and the frequency of such behavior.

\section{Conclusion}

In the present study, it has been shown that the acceptance of food offerings was the most influential impeding factor to adherence with weight loss diets in weight regainers. Moreover, the acceptance of food offerings was related to lower self-esteem. The fact that a vast majority 
of dieting people regain the weight they lost may be reflective of the fact that the attractive food cues inherent in food offerings makes adherence with caloric restriction extremely difficult for some individuals. Findings from this study can be used to improve traditional weightloss programs by the addition of behavioral interventions that focusing on improving self-esteem and shifting individual responses to food offerings and to other socially motivating reinforcers.

\section{Acknowledgments}

The authors have nothing to disclose. Supported in part by the Intramural Research Program of the $\mathrm{NIH}, \mathrm{NIDDK}$. No additional outside funding/support was received for this study.

\section{References}

1. Byrne S, Cooper Z, Fairburn C (2003) Weight maintenance and relapse in obesity: a qualitative study. Int J Obes Relat Metab Disord 27: 955-962.

2. Wilson GT (2002) Eating disorders and obesity: a comprehensive handbook. (2ndedn), New York: Guilford Press

3. Dansinger ML, Gleason JA, Griffith JL, Selker HP, Schaefer EJ (2005) Comparison of the Atkins, Ornish, Weight Watchers, and Zone diets for weight loss and heart disease risk reduction: a randomized trial. JAMA 293: 43-53.

4. Leibel RL, Rosenbaum M, Hirsch J (1995) Changes in energy expenditure resulting from altered body weight. N Engl J Med 332: 621-628.

5. Tsai AG, Wadden TA (2005) Systematic review: an evaluation of major commercial weight loss programs in the United States. Ann Intern Med 142 56-66.

6. Polivy J, Herman CP, Coelho JS (2008) Caloric restriction in the presence of attractive food cues: external cues, eating, and weight. Physiol Behav 94: 729-

7. Brownell KD, Horgen KB (2004) Food fight. New York: McGraw Hill.

8. Fedoroff I, Polivy J, Herman CP (2003) The specificity of restrained versus unrestrained eaters' responses to food cues: general desire to eat, or craving for the cued food? Appetite 41: 7-13.

9. Fedoroff IC, Polivy J, Herman CP (1997) The effect of pre-exposure to food cues on the eating behavior of restrained and unrestrained eaters. Appetite 28: 33-47.

10. Cornell CE, Rodin J, Weingarten H (1989) Stimulus-induced eating when satiated. Physiol Behav 45: 695-704.
11. Rozin P, Dow S, Moscovitch M, Rajaram S (1998) What causes humans to begin and end a meal? A role for memory for what has been eaten, as evidenced by a study of multiple meal eating in amnesic patients. The journals of gerontology Series B, Psychological sciences and social sciences 9: 392396

12. Tetley A, Brunstrom J, Griffiths P (2009) Individual differences in food-cue reactivity. The role of $\mathrm{BMI}$ and everyday portion-size selections. Appetite 52 614-620.

13. Greenberg I, Perna F, Kaplan M, Sullivan MA (2005) Behavioral and psychological factors in the assessment and treatment of obesity surgery patients. Obes Res 13: 244-249.

14. Wardle J, Cooke L (2005) The impact of obesity on psychological well-being Best Pract Res Clin Endocrinol Metab 19: 421-440.

15. Mauro M, Taylor V, Wharton S, Sharma AM (2008) Barriers to obesity treatment. Eur J Intern Med 19: 173-180.

16. Shapurian R, Hojat M, Nayerahmadi H (1987) Psychometric characteristics and dimensionality of a Persian version of Rosenberg Self-esteem Scale. Percept Mot Skills 65: 27-34.

17. Rosenberg M (1979) Conceiving the Self. (6th edn), Basic Books, New York.

18. Leone $T$, Pliner $P$, Peter Herman $C$ (2007) Influence of clear versus ambiguous normative information on food intake. Appetite 49: 58-65.

19. Vartanian LR, Herman CP, Wansink B (2008) Are we aware of the external factors that influence our food intake? Health Psychol 27: 533-538.

20. Egecioglu E, Skibicka KP, Hansson C, Alvarez-Crespo M, Friberg PA, et al. (2011) Hedonic and incentive signals for body weight control. Rev Endocr Metab Disord 12: 141-151.

21. Nunn RG, Newton KS, Faucher P (1992) 2.5 years follow-up of weight and Body Mass Index values in the Weight Control for Life! program: a descriptive analysis. Addict Behav 17: 579-585.

22. Pekkarinen T, Mustajoki $P$ (1997) Comparison of behavior therapy with and without very-low-energy diet in the treatment of morbid obesity. A 5-year outcome. Arch Intern Med 157: 1581-1585.

23. Torgerson JS, Lissner L, Lindroos AK, Kruijer H, Sjöström L (1997) VLCD plus dietary and behavioural support versus support alone in the treatment of severe obesity. A randomised two-year clinical trial. Int J Obes Relat Metab Disord 21 987-994.

24. Rastmanesh R, Gluck ME, Shadman Z (2009) Comparison of body dissatisfaction and cosmetic rhinoplasty with levels of veil practicing in Islamic women. Int J Eat Disord 42: 339-345. 